www.nature.com/pj

\title{
Preparation of 20-nm poly(styrene derivative) particles via polymerization with nonionic surfactant at the phase-inversion temperature
}

\author{
Kiyoshi Suzuki, Kohei Nishiyama, Isao Yamanaka, Takatoshi Koshiba and Shuzaemon Satoh \\ Several types of styrene monomer derivatives were polymerized in monomer, water, polyoxyethylene alkyl phenyl ether and \\ initiator mixtures at various temperatures to form small particles. Smaller particles were obtained at higher radical generation \\ rates at the phase-inversion temperature (PIT). Large coagula or secondary particles were obtained at temperatures higher than \\ the PIT. The average diameter of the polymer particles obtained via the polymerization of $p$-methylstyrene and styrene at the \\ PIT was smaller than those of divinylbenzene and 4-t-butylstyrene. Poly( $p$-methylstyrene) particles, which had an average \\ diameter of $21 \mathrm{~nm}$ and a polymer weight fraction of $5 \%$, were prepared. \\ Polymer Journal (2012) 44, 1077-1081; doi:10.1038/pj.2012.75; published online 16 May 2012
}

Keywords: emulsion polymerization; nanometer-sized particles; nonionic surfactant; particle-size distribution; phase-inversion temperature; polystyrene

\section{INTRODUCTION}

The preparation of nanometer-sized polymer particles with a narrow size distribution, which can be utilized in nanoscale devices, is desirable. Polymer particles with an average diameter $<10 \mathrm{~nm}$ and with a narrow size distribution were reported to be prepared by polymerizing the specific surface-active monomer, didecyldimethylammounium methacrylate. ${ }^{1}$ However, polymer particles that have an average diameter $<10 \mathrm{~nm}$ and a narrow size distribution have not been obtained by polymerizing either styrene or its derivatives.

Ferrick et al. ${ }^{2}$ reported that polystyrene particles with an average diameter of $10.8 \mathrm{~nm}$ were prepared using photoinitiated microemulsion polymerization with $2,2^{\prime}$-azobisisobutyronitrile as an initiator and cetyltrimethylammonium bromide as a surfactant; however, the diameter of the particles was estimated from results obtained with a transmission electron microscope and was not confirmed. Furthermore, the weight ratio of polymer to surfactant is less than one-third. Antonietti et al. ${ }^{3}$ succeeded in controlling the diameter of the generated polystyrene particles by changing the molar ratio of surfactant to charged monomer. They successfully prepared the particles and the hydrodynamic diameter that was measured by dynamic light scattering (DLS) was as small as $20 \mathrm{~nm}$ when using cetyltrimethylammonium bromide. The reported average diameter of the polystyrene particle, as measured by small-angle neutron scattering, was $11.0 \mathrm{~nm}$.

The polymer particles that are prepared and stabilized with ionic surfactants in the aqueous phase lose their stability if salts are added. A nonionic surfactant, such as polyoxyethylene alkyl ether, may be a substitution candidate for ionic surfactants. However, nonionic surfactants usually have weaker stabilization effects for polymer particles, and the polymer particles that are obtained using a nonionic surfactant tend to grow larger than those obtained using an ionic surfactant. ${ }^{4}$ Polystyrene particles with average diameters of $<30 \mathrm{~nm}$ have not been prepared without the use of ionic surfactants.

Nevertheless, nonionic surfactants show an extraordinary behavior that is not exhibited by ionic surfactants: temperature-induced phase inversion. A certain mixture of a nonionic surfactant with more than two immiscible solvents, such as oil and water, generates an oil-in-water $(\mathrm{O} / \mathrm{W})$ emulsion at temperatures lower than the so-called phase-inversion temperature (PIT), whereas it generates a water-in-oil (W/O) emulsion at temperatures higher than the PIT. The surface tension between the oil-rich phase and the water-rich phase reaches a minimum value at the PIT, and small miniemulsion droplets can be generated by gentle agitation at the PIT. Spernath and Magdassi ${ }^{5}$ reported that small miniemulsion droplets were generated from a rapid decrease in temperature, past the PIT, to a temperature much lower than the PIT. Saito and Shinoda ${ }^{6}$ reported that good stability against an increase in the average diameter of the generated miniemulsion droplets is achieved at temperatures that are $>20^{\circ} \mathrm{C}$ below the PIT. There have been some reports of the preparation of small polymer particles from polymerization in miniemulsions that are generated by utilizing the PIT. ${ }^{7}$ Polymerization in miniemulsions that are generated by utilizing the PIT is more effective than microemulsion and emulsion polymerization for the generation of small polymer particles by polymerizing a monomer that has very low 
water solubility and for easily incorporating water-insoluble molecules, such as pyrene, into the generated polymer particles. However, the average diameter of the generated polymer particles is $>50 \mathrm{~nm}^{7}$

In this study, polymer particles with an average diameter $<30 \mathrm{~nm}$ were prepared for the first time from the direct polymerization of a styrene monomer derivative without ionic surfactants with a nonionic surfactant. The effects of initiator concentration, temperature, surfactant type and monomer type on the average diameter of the generated polymer particles are reported.

\section{MATERIALS AND METHODS/EXPERIMENTAL PROCEDURE Materials}

Styrene, $p$-methylstyrene, divinylbenzene and 4-t-butylstyrene monomer were purchased from Wako Pure Chemical Industries, Ltd. (Osaka, Japan) and were purified using the following method. One part of the monomer was washed three times using one-third part of aqueous potassium hydroxide solution (15 wt.\%) to remove hydrophilic impurities and was then washed three times using one-third part of pure water to remove the residual potassium hydroxide. Then, the washed monomer was distilled under a nitrogen atmosphere under reduced pressure and stored in a refrigerator until use. The temperatures used during the distillation were $45^{\circ} \mathrm{C}$ for styrene, $60^{\circ} \mathrm{C}$ for $p$-methylstyrene, $85^{\circ} \mathrm{C}$ for divinylbenzene and $95^{\circ} \mathrm{C}$ for 4 -t $t$-butylstyrene. Polyoxyethylene nonyl phenyl ether, with an average number of oxyethylene units of approximately 20 (N200), was supplied by Sanyo Chemical Industries, Ltd (Kyoto, Japan) and used without further purification. Polyoxyethylene octyl phenyl ether, with an average number of oxyethylene units of approximately 10 (N100), methanol (guaranteed reagent; GR), chloroform, hexadecane (GR), hydroquinone, potassium persulfate (KPS, GR), ferrous sulfate $\left(\mathrm{FeSO}_{4}, \mathrm{GR}\right)$ and rongalite $\mathrm{C}$ (sodium formaldehyde sulfoxylate, GR) were purchased from Wako Pure Chemical Industries, Ltd and were used without further purification. Water used was purified using a Milli-Q Simplicity UV (Merck KGaA, Darmstadt, Germany). Sodium dodecyl sulfate, which was used as special reagent for the analysis of insoluble protein, was purchased from Nacalai tesque, Inc. (Kyoto, Japan) and was used as received. $2,2^{\prime}$-azobisisobutyronitrile was purchased from Wako Pure Chemical Industries, Ltd., purified by recrystallization in chloroform by adding methanol, vacuum-dried under reduced pressure to remove the residual methanol and stored in a refrigerator until use. Nitrogen gas (99.999\%) was purchased from Sumitomo Seika Chemicals Co., Ltd (Osaka, Japan).

\section{Determination of the PIT}

A certain amount of monomer that contained a certain amount of hydroquinone or a certain amount of unpurified monomer (that is, involving radical polymerization inhibitor, as supplied) is mixed with a certain amount of the nonionic surfactant and water and then loaded into a vessel. Typically, the weight of the mixed nonionic surfactant is the same as that of the mixed monomer, and the weight of the mixed water is 18 times greater than that of the mixed monomer. The vessel is dipped into a thermostatted bath and gently agitated. The transparency is checked visually. The transparency check is repeated with the thermostatted bath at various temperatures. At temperatures lower than a certain temperature, the transparency increases as the temperature approaches the certain temperature. The turbidity of the mixture abruptly increased as the temperature was increased above the certain temperature. The temperature at which the transparency reaches its maximum value is regarded as the PIT of the mixture.

\section{Polymerization}

Small miniemulsion droplets were reported to be generated by a rapid decrease in temperature past the PIT. ${ }^{5}$ The stability of the generated miniemulsion droplets is reported to be good at temperatures $>20^{\circ} \mathrm{C}$ below the PIT. ${ }^{6}$ Nevertheless, preliminary studies to determine a suitable temperature profile for the generation of smaller particles during and/or before polymerization have revealed that the direct polymerization at the PIT with gentle agitation generates smaller particles than polymerization at a temperature $>20^{\circ} \mathrm{C}$ below the PIT after decreasing the temperature rapidly past the PIT.

If the polymerization temperature was higher than room temperature $\left(25-30^{\circ} \mathrm{C}\right)$, only KPS was used as an initiator. If the polymerization temperature was lower than room temperature, redox initiation was performed. In the experiment in which only KPS was used as an initiator, a measured amount of KPS and $0.50 \mathrm{~g}$ of surfactant were dissolved in $9.0 \mathrm{~g}$ of water; this solution is regarded as mixture $\mathrm{A}$. In the experiment in which redox initiators were used, $0.035 \mathrm{~g}$ of ferrous sulfate, $0.035 \mathrm{~g}$ of rongalite $\mathrm{C}$ and $0.50 \mathrm{~g}$ of surfactant were dissolved in $8.0 \mathrm{~g}$ of water and then $0.50 \mathrm{~g}$ monomer was added; this mixture was regarded as mixture A. Nitrogen gas was bubbled through mixture A for $>30 \mathrm{~min}$ in a small glass vessel equipped with a magnetic stirrer, an inlet of nitrogen gas and an outlet for sampling. The vessel was thermostatted at approximately $0{ }^{\circ} \mathrm{C}$ by dipping the vessel into an ice and water mixture bath during bubbling. After bubbling, the small vessel was sealed. In the experiment in which only KPS was used as an initiator, $0.50 \mathrm{~g}$ of monomer was injected into the sealed vessel using a syringe through a septum. In the experiment in which redox initiators were used, $1.0 \mathrm{~g}$ of an aqueous solution containing $0.017 \mathrm{~g}$ of KPS was injected into the vessel using a syringe through a septum. Then, the injected vessel was dipped into a thermostatted bath to regulate the temperature to the required polymerization temperature to begin polymerization. During the polymerization, the mixture in the vessel was agitated by a magnetic stirrer with a rotation speed of 90 r.p.m. After a certain period of time for the polymerization, the vessel was removed from the thermostatted bath and exposed to air to stop polymerization. In the experiment in which redox initiators were used, two or three drops of a hydroquinone aqueous solution (1000 p.p.m.) were added to stop polymerization.

\section{Analysis}

Most of the obtained sample was withdrawn from the vessel and dried at room temperature for more than a half day and then dried at $65^{\circ} \mathrm{C}$ for more than 1 day. The monomeric conversion was calculated by subtracting the total weight of the surfactant and the initiators estimated to exist in the withdrawn sample, assuming that the sample was homogeneously withdrawn. Another portion of the obtained sample was diluted with a large amount of pure water and the average diameter and particle-size distribution were measured using DLS (DLS-7000; Otsuka Electronics Co., Ltd., Hirakata, Japan) and transmission electron microscopy (TEM, H-700A; Hitachi High-Technologies Co., Tokyo, Japan). For the TEM measurements, the diluted samples were sprayed onto a grid mesh that was covered with a collodion film coated with vapordeposited carbon film, and then, the grid mesh was coated using vapor deposition of platinum and vanadium for shadowing.

\section{RESULTS AND DISCUSSION}

\section{PITs}

The determined PITs are listed in Table 1. For all the monomers used, the PITs with $\mathrm{N} 200$ are approximately $40^{\circ} \mathrm{C}$ higher than those with N100. The PITs are higher for the monomers in the following order: 4- $t$-butylstyrene $>$ divinylbenzene $\sim p$-methylstyrene $>$ styrene; this order

\section{Table 1 Determined phase-inversion temperatures}

\begin{tabular}{lclc}
\hline Monomer $^{\mathrm{a}}$ & Surfactant $^{\mathrm{b}}$ & PIT $\left.{ }^{\circ} \mathrm{C}\right)$ & Log Pow $^{\mathrm{c}}$ \\
\hline Styrene & $\mathrm{N} 200$ & 53 & $3.0^{8}$ \\
Styrene & $\mathrm{N} 100$ & 10 & $3.0^{8}$ \\
$p$-Methylstyrene & $\mathrm{N} 200$ & 60 & $3.58^{8}$ \\
$p$-Methylstyrene & $\mathrm{N} 100$ & 20 & $3.58^{8}$ \\
Divinylbenzene & $\mathrm{N} 200$ & 60 & $3.59^{8}$ \\
Divinylbenzene & $\mathrm{N} 100$ & 22.5 & $3.59^{8}$ \\
4-t-Butylstyrene & $\mathrm{N} 200$ & 83 & $4.5^{9}$ \\
4-t-Butylstyrene & $\mathrm{N} 100$ & 45 & $4.5^{9}$ \\
\hline
\end{tabular}

Abbreviation: PIT, phase-inversion temperature.

a Monomer weight ratio to water is $5 / 90$.

bSurfactant weight ratio to water is $5 / 90$.

cPow is partition coefficient of the monomer between $n$-octanol and water phases. 
may reflect the hydrophobicity of the monomers. The degree of hydrophobicity is related to the partition coefficient between the $n$-octanol and water phases (Pow).

\section{Effect of radical generation rate}

A faster radical generation rate has been reported to be suitable for the generation of smaller polymer particles; ${ }^{10}$ the suitability of this higher rate was checked first. First, the time required for the monomeric conversion to reach $>80 \%$ is determined, and the dependency of the average diameter of the obtained polymer particles on the reaction time is studied. Styrene and N200 were used as the monomer and nonionic surfactant, respectively. The amount of KPS loaded into the reaction was fixed at $0.24 \mathrm{~g}$. The polymerization was performed at the PIT $\left(53^{\circ} \mathrm{C}\right)$. The measured monomeric conversion, the number-average diameter of the observed particles, which was measured from the TEM images, and the average diameter measured with DLS at 15 and $60 \mathrm{~min}$ are shown in Table 2. The conversion approaches no less than 0.97 at $15 \mathrm{~min}$. A typical TEM image for the sample after $60 \mathrm{~min}$ is shown in Figure 1. The generated polystyrene particles are spherical and the size distribution is polydisperse. The observed particles for all of the samples obtained in this study are spherical. Both of the average diameters that were measured with TEM and DLS are almost constant, irrespective of the polymerization time. The variation of approximately $3 \mathrm{~nm}$ may have resulted from experimental errors. At the DLS measurements, the average diameter $\left(D_{\text {DLS }}\right)$ of the spherical particles is calculated using Equation (1), and the diameters must be larger than the numberaverage diameter, which is in good agreement with the obtained experimental results. In Equation (1), $D_{i}$ is the diameter of $i$-th particle and the summation is over all of the particles.

$$
D_{\text {DLS }}=\frac{\sum_{i} D_{i}^{6}}{\sum_{i} D_{i}^{5}}
$$

The coefficient of variation (CV), which is defined as the s.d. divided by the number-average diameter, is calculated from the particle-size distribution measured from the TEM images and is almost constant, irrespective of time, as shown in Table 2. In conclusion, under these experimental conditions, the conversion reaches no less than 0.97 at $15 \mathrm{~min}$, and the average diameter and $\mathrm{CV}$ value do not change with time at a conversion as high as 0.97 .

Therefore, the average diameter and CV values of the polystyrene particles that were prepared with N200 and KPS at a reaction time of 60 min using different concentrations of KPS were measured with TEM and DLS and are shown in Table 2 along with the conversion

Table 2 Effect of reaction time and KPS concentration on average diameter and $\mathrm{CV}$ values of polystyrene particles prepared by polymerization with $0.5 \mathrm{~g} \mathrm{~N} 200,0.5 \mathrm{~g}$ styrene and $9 \mathrm{~g}$ water at $53^{\circ} \mathrm{C}$

\begin{tabular}{lccccc}
\hline $\begin{array}{l}\text { Reaction time } \\
(\min )\end{array}$ & $\begin{array}{c}\text { KPS } \\
(g)\end{array}$ & $\begin{array}{c}\mathrm{D}_{n} \\
(\mathrm{~nm})^{\mathrm{a}}\end{array}$ & $\begin{array}{c}\text { CV (TEM) } \\
(-)\end{array}$ & $\begin{array}{c}\mathrm{D}_{\text {DLS }} \\
(\mathrm{nm})\end{array}$ & $\begin{array}{c}\text { Conversion } \\
(-)\end{array}$ \\
\hline 15 & 0.24 & 23.9 & 0.35 & 32.6 & 0.97 \\
60 & 0.24 & 20.9 & 0.33 & 30.9 & 1.0 \\
60 & 0.24 & 21.2 & 0.33 & 31.1 & 0.97 \\
60 & 0.08 & 25.1 & 0.25 & 31.1 & 1.0 \\
60 & 0.027 & 29.4 & 0.24 & 38.0 & 0.94 \\
60 & 0.027 & 26.1 & 0.25 & 38.1 & 0.88
\end{tabular}

Abbreviations: CV, coefficient of variation; DLS, dynamic light scattering; KPS, potassium persulfate; TEM, transmission electron microscopy.

a Number-average diameter measured with TEM. value. The conversions for all of the experiments shown in Table 2 are close to unity. To check the reproducibility, the polymerization with KPS amounts of 0.24 and $0.027 \mathrm{~g}$ was performed twice. The reproducibility is not bad, although the measured average diameter varied by $3.3 \mathrm{~nm}(12 \%)$ for TEM with $0.027 \mathrm{~g}$ of KPS. Both the number-average diameter $\left(D_{\mathrm{n}}\right)$ that was measured using TEM and the average diameter measured using DLS ( $D_{\text {DLS }}$ ) decreased with increasing concentrations of KPS, in other words, with the increase in the radical generation rate.

Figure 2 shows a plot of the average diameters against the concentration of KPS. The dependency of the $D_{\mathrm{n}}$ value on the KPS concentration is -0.13 and the dependency of $D_{\text {DLS }}$ is -0.094 . The number of polymer particles generated $(N)$ holds Equation (2).

$$
M X_{\mathrm{m}}(1+\gamma)=\rho_{\mathrm{P}} N \pi d^{3} / 6
$$

where $M$ is charged amount of monomer $(\mathrm{g}), X_{\mathrm{m}}$ is the conversion value and and $d$ is volume-average (mean-volume) diameter $(\mathrm{cm})$, which may be approximated by other average diameters. $\gamma$ is the volume ratio of the monomer to polymer in the polymer particles, which is approximated as

$$
\gamma=\left(M_{\mathrm{P}} / \rho_{\mathrm{M}}\right) /\left(P_{\mathrm{P}} / \rho_{\mathrm{P}}\right)
$$

where $M_{\mathrm{P}}$ and $P_{\mathrm{P}}$ are the amounts of monomer and polymer in the whole polymer particles, respectively. $P_{\mathrm{p}}$ equals $M X_{\mathrm{m}} \cdot \rho_{\mathrm{M}}$ and $\rho_{\mathrm{P}}$ are

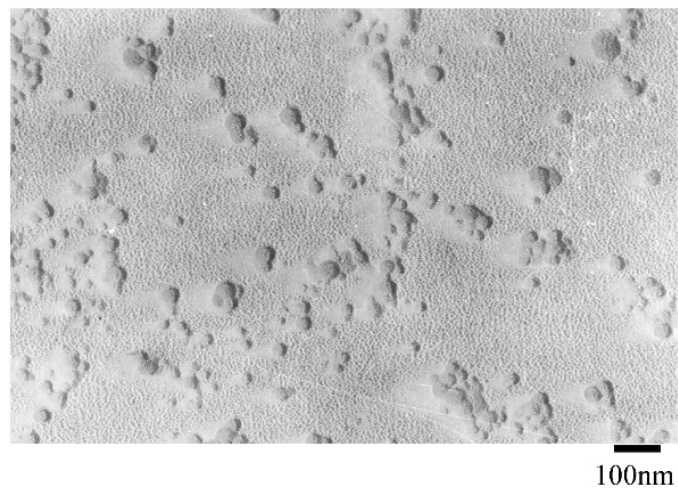

Figure 1 Typical TEM image of the polymer particles obtained by styrene polymerization for $60 \mathrm{~min}$ with $\mathrm{N} 200$ and KPS $(0.24 \mathrm{~g}$ per $9 \mathrm{~g}$ water) at $53^{\circ} \mathrm{C}$.

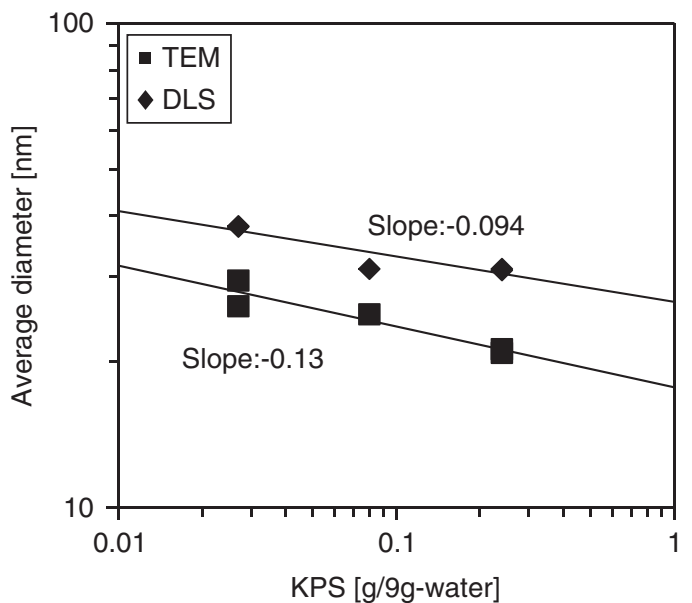

Figure 2 Effect of the KPS concentration on the average diameter of the polystyrene particles obtained by polymerization for $60 \mathrm{~min}$ with N200 and KPS at $53^{\circ} \mathrm{C}$. 
the densities $\left(\mathrm{g} \mathrm{cm}^{-3}\right)$ of the monomer and polymer, respectively. For all of the experiments shown in Figure 2, $M$ is constant $(0.5 \mathrm{~g}), X_{\mathrm{m}}$ is close to unity and $\gamma$ is almost 0 . Therefore, the number of polymer particles generated, $N$, is proportional to the reciprocal of the third power of the average diameter, $d$, and is proportional to the $(-0.094) \times(-3)=0.28$ to $(-0.13) \times(-3)=0.39$ power of the initiator (KPS) concentration (see Supplementary Information S1). The 0.4th power dependency of the number of polymer particles generated on the initiator concentration (radical generation rate) is predicted by the Smith-Ewart Theory ${ }^{11}$ assuming the following:

(1) Radical entry into a micelle generates a polymer particle, and the rate of volume increase for each of the generated polymer particles is constant.

(2) The whole radical entry rate into the micelles per unit volume of the reaction system is almost constant, irrespective of the reaction time until the generation of particles ends.

(3) The generation of particles ends when the total surface area of the generated polymer particles equals the maximum surface area that the charged surfactant can cover and stabilize.

The miniemulsified monomer droplets that were generated during the polymerization experiments may behave like micelles. The observed dependency value $(0.28-0.39)$ is close to 0.4 , which may indicate that the mechanism follows assumptions (1) through (3) and takes place during the polymerization experiments, although another mechanism is not ruled out.

Effect of the polymerization temperature compared with the PIT Styrene polymerization was performed using $0.5 \mathrm{~g}$ of styrene monomer, $0.5 \mathrm{~g}$ of $\mathrm{N} 200,0.24 \mathrm{~g}$ of KPS and $9 \mathrm{~g}$ of water at various temperatures around the PIT, $53{ }^{\circ} \mathrm{C}$, to study the effects of the temperature on the average diameter of the generated polymer particles. The reaction time was fixed at $60 \mathrm{~min}$. The average diameters that were measured using TEM and DLS are shown in Figure 3 . The average diameter of the generated particles obtained at the PIT $\left(53^{\circ} \mathrm{C}\right)$ is less than those obtained at lower $\left(33^{\circ} \mathrm{C}\right)$ and higher $\left(67^{\circ} \mathrm{C}\right)$ temperatures (Supplementary Information S2). At temperatures greater than the PIT $\left(67^{\circ} \mathrm{C}\right)$, large aggregations (secondary particles) of small particles (primary particles) are observed. In Figure 3, the average diameter of the primary particles is shown as a square (TEM). The average diameter measured using DLS drastically increases at temperatures greater than the PIT, whereas the average diameter of the primary particles obtained at $67^{\circ} \mathrm{C}$ and measured using TEM is not much larger than that obtained at the PIT. This difference may be attributed to the instability of the
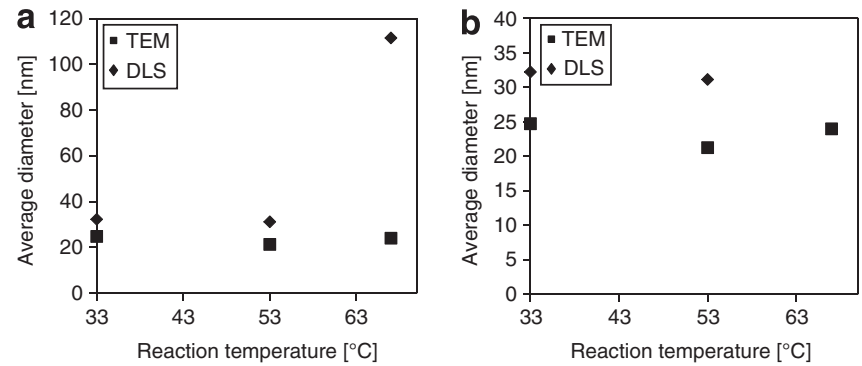

Figure 3 Effect of the reaction temperature on the average diameter of the polystyrene particles obtained by (a) 60 min of polymerization with $0.5 \mathrm{~g}$ N200, $0.5 \mathrm{~g}$ styrene, $0.24 \mathrm{~g}$ KPS and $9 \mathrm{~g}$ water. (b) is enlarged: the maximum particle diameter is $40 \mathrm{~nm}$. hydrophobic domain in the aqueous phase at temperatures greater than the PIT. The hydrophobic domains cannot be dispersed into the aqueous phase but rather coagulate to form the aggregations (secondary particles) at temperatures greater than the PIT.

Figure 4 shows the $\mathrm{CV}$ value of the primary particles that were measured using TEM, which decreases as the reaction temperature increases, possibly because of the difficulty in counting small particles among the aggregated particles.

In summary, the diameter of the particles reaches its minimum value around the PIT, and large aggregations (secondary particles) are formed at temperatures greater than the PIT. Thus, polymerization at the PIT is suitable for the preparation of small particles.

\section{Effect of monomer and surfactant type}

The average diameters that were measured using TEM and DLS and the $\mathrm{CV}$ values of the polymer particles that were prepared by polymerization for $>30 \mathrm{~min}$ at the PIT using various types of monomers and two types of nonionic surfactants are listed in Table 3. In the cases where the PIT is $>30^{\circ} \mathrm{C}$, the polymerization was initiated using a high concentration of KPS $(0.24 \mathrm{~g}$ per $9 \mathrm{~g}$ water $)$, and the monomeric conversion is almost $100 \%$; conversions of $>100 \%$ possibly result from experimental errors such as sample

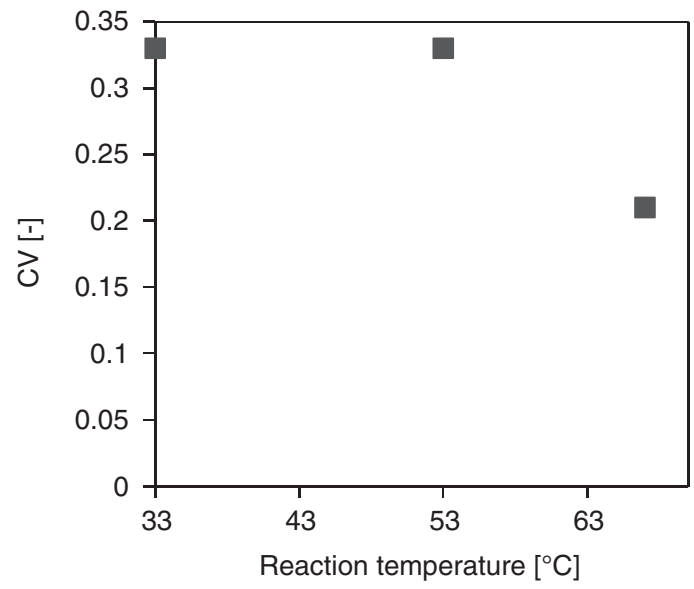

Figure 4 Effect of the reaction temperature on the CV of the primary polystyrene particles obtained by 60 min of polymerization with $0.5 \mathrm{~g} \mathrm{~N} 200$, $0.5 \mathrm{~g}$ styrene, $0.24 \mathrm{~g}$ KPS and $9 \mathrm{~g}$ water.

Table 3 Effect of types of monomers and surfactants on the properties of the polymer particles prepared by polymerization at PIT

\begin{tabular}{|c|c|c|c|c|c|c|c|}
\hline \multirow[b]{3}{*}{ Surfactant } & \multirow[b]{3}{*}{ Monomer } & \multirow{2}{*}{$\begin{array}{c}D^{b} \\
(T E M)\end{array}$} & \multicolumn{3}{|c|}{$D^{c}$} & \multirow[b]{2}{*}{$\log$} & \multirow[b]{2}{*}{ PIT } \\
\hline & & & $C V(T E M)$ & $(D L S)$ & Conversion & & \\
\hline & & $(n m)$ & $(-)$ & $(n m)$ & $(-)$ & Pow & $\left({ }^{\circ} \mathrm{C}\right)$ \\
\hline \multirow[t]{4}{*}{ N200 } & Styrene & 21.0 & 0.33 & 31.0 & 0.99 & $3.0^{8}$ & 53 \\
\hline & p-Methylstyrene & 20.3 & 0.30 & 28.1 & 1.00 & $3.58^{8}$ & 60 \\
\hline & Divinylbenzene & 36.5 & 0.28 & 91.0 & 1.08 & $3.59^{8}$ & 60 \\
\hline & 4-t-Butylstyrene & 24.2 & 0.32 & 76.1 & 1.03 & $4.5^{9}$ & 83 \\
\hline \multirow[t]{4}{*}{ N100 } & Styrene & 29.2 & 0.24 & 37.2 & 0.64 & $3.0^{8}$ & 10 \\
\hline & p-Methylstyrene & 32.8 & 0.49 & 51.6 & 0.27 & $3.58^{8}$ & 20 \\
\hline & Divinylbenzene & 23.4 & 0.49 & 121.4 & 0.87 & $3.59^{8}$ & 22.5 \\
\hline & 4-t-Butylstyrene & 25.4 & 0.29 & 28.1 & 1.00 & $4.5^{9}$ & 45 \\
\hline
\end{tabular}

Abbreviations: DLS, dynamic light scattering; PIT, phase-inversion temperature; TEM, transmission electron microscopy.

apolymerization period is $60 \mathrm{~min}$ for styrene and $30 \mathrm{~min}$ for the other monomers.

bNumber-average diameter measured with TEM.

cAverage diameter measured with DLS. 
inhomogeneity (coagulation and precipitation). In the cases where the PIT is $<30^{\circ} \mathrm{C}$, the polymerization was initiated using low concentration of KPS $(0.017 \mathrm{~g}$ per $9 \mathrm{~g}$ water), ferrous sulfate $(0.035 \mathrm{~g}$ per $9 \mathrm{~g}$ water) and rongalite $\mathrm{C}(0.035 \mathrm{~g}$ per $9 \mathrm{~g}$ water $)$; the anionic surfactant sodium dodecyl sulfate was added to the reaction mixture just after the end of the polymerization to stabilize the generated particles at room temperature.

In the cases where the PIT is $<30{ }^{\circ} \mathrm{C}$, the monomeric conversion is $<90 \%$. The average diameter of the primary particles, as measured using TEM, has no clear relationship with the reaction temperature (that is, with the partition coefficient, Pow) and the hydrophobicity of monomer. On the other hand, the average diameter measured using DLS increases as the reaction temperature increases; the reason for this relationship is not clear.

With N200, the average diameter of p-methylstyrene, whose Pow is higher than styrene, is slightly lower than that of styrene. The smaller diameter of $p$-methylstyrene may be because of the restricted diffusion of the less water-soluble monomer in the aqueous phase between the miniemulsions during polymerization, that is, the restriction of Ostwald ripening. However, less water-soluble monomers, such as divinylbenzene and 4- $t$-butylstyrene, yield larger particles than styrene; therefore, the obtained results cannot be explained only by Ostwald ripening. The diameters measured using DLS with divinylbenzene and 4-t-butylstyrene are by far larger than those measured using TEM, which reflects the aggregation and/or coagulation of the primary particles. Divinylbenzene has two vinyl groups per molecule and the generated polymer particles with residual vinyl groups can be connected to each other and tend to aggregate (flocculate). The PIT of 4-t-butylstyrene is much higher than that of styrene, $p$-methylstyrene or divinylbenzene. At higher temperatures, the thermal motion of the generated polymer particles is greater; thus, coagulation of the particles is promoted. For 4-t-butylstyrene, the PIT with $\mathrm{N} 100\left(45^{\circ} \mathrm{C}\right)$ is lower than that with $\mathrm{N} 200\left(83^{\circ} \mathrm{C}\right)$. The diameters of the polymer particles obtained with 4 -t-butylstyrene and N100, measured using DLS, are not much larger than those measured using TEM. Therefore, the polymerization of the mixture of $p$ methylstyrene and N200 with a moderately high PIT value without two vinyl groups yields the smallest polymer particles.

For N100, the average diameter that was measured using DLS for the particles obtained using redox initiators (KPS, ferrous sulfate and rongalite $\mathrm{C}$ ), that is, with styrene, $p$-methylstyrene and divinylbenzene, is larger than those obtained using only KPS, that is, with 4-tbutylstyrene. The procedure using redox initiators in this study may not be adequate for the preparation of small particles. Further studies to search for a better initiator system are desired.

In summary, the effects that the radical generation rate, temperature and monomer and surfactant type had on the average diameter of the polymer particles that were generated by polymerization using nonionic surfactant polyoxyethylene alkyl phenyl ether and water were studied. The higher the radical generation rate, the smaller the diameter of the polymer particles generated. At temperatures higher than the PIT, aggregation or coagulation of the primary particles occurs and large secondary particles or coagula are produced. The average particle diameter reaches its minimum value at the PIT. The polymerization of $p$-methylstyrene, which has a PIT that is slightly higher than that of styrene and a partition coefficient between $n$-octane and water (Pow) that is higher than that of styrene, yields slightly smaller particles than polymerization with styrene. However, the polymerization of 4-t-butylstyrene, which has a PIT that is much higher and a Pow that is much larger than that of $p$-methylstyrene, yields larger particles, which may be due to aggregation or coagulation. The polymerization of divinylbenzene, which has two vinyl groups, also results in aggregation. $p$-Methylstyrene particles, which have an average diameter of $21 \mathrm{~nm}$ and a polymer weight fraction of approximately $5 \%$, are successfully prepared.

\section{ACKNOWLEDGEMENTS}

This study was financially supported by a Grant-in-Aid for Scientific Research, the Ministry of Education, Culture, Sports, Science and Technology, Japan (Grant-in-Aid 20710106). Polyoxyethylene nonyl phenyl ether with an average number of oxyethylene units of approximately 20 (N200) was supplied by Sanyo Chemical Industries, Ltd.

1 Hammouda, A., Gulik, T. \& Pileni, M. P. Synthesis of nanosize latexes by reverse micelle polymerization. Langmuir 11, 3656-3659 (1995).

2 Ferrick, M. R., Murtagh, J. \& Thomas, J. K. Synthesis and characterization of polystyrene latex particles. Macromolecules 22, 1517-1517 (1989).

3 Antonietti, M., Bremser, W., Mueschenborn, D., Rosenauer, C., Schupp, B. \& Schmidt, M. Synthesis and size control of polystyrene latices via polymerization in microemulsion. Macromolecules 24, 6636-6643 (1991).

4 Suzuki, K., Wakatuki, Y., Shirasaki, S., Fujita, K., Kato, S. \& Nomura, M. Effect of mixing ratio of anionic and nonionic emulsifiers on the kinetic behavior of methyl methacrylate emulsion polymerization. Polymer 46, 5890-5895 (2005).

5 Spernath, L. \& Magdassi, S. Formation of silica nanocapsules from nanoemulsions obtained by the phase inversion temperature method. Micro. Nano. Lett. 5, 28-36 (2010).

6 Saito, H. \& Shinoda, K. The stability of W/O type emulsions as a function of temperature and of the hydrophilic chain length of the emulsifier. J. Colloid. Interf. Sci. 32, 647-651 (1970).

7 Spernath, L. \& Magdassi, S. A. New method for preparation of poly-lauryl acrylate nanoparticles from nanoemulsions obtained by the phase inversion temperature process. Polym. Adv. Technol. 18, 705-711 (2007).

8 International Labour Organization, International Chemical Safety Cards. http://www.ilo. org/dyn/icsc/showcard.home (May, 2012).

9 Molinspiration Cheminformatics, Calculation of Molecular Properties and Bioactivity Score. http://www.molinspiration.com/cgi-bin/properties (August, 2011).

10 Antonietti, M. \& Landfester, K. Polyreactions in miniemulsions. Prog. Polym. Sci. 27, 689-757 (2002).

11 Smith, W.V. \& Ewart, R.H. Kinetics of emulsion polymerization. J. Chem. Phys. 16, 592-599 (1948).

Supplementary Information accompanies the paper on the Polymer Journal website (http://www.nature.com/pj) 